\title{
Adsorption of Polar Volatile Organic Compounds by a Crystalline Network Structure Based on a Bis(benzimidazole) $\mathrm{NiCl}_{2}$ Complex
}

Shun Ohta, ${ }^{* \dagger}$ Yurika Iwabuchi, ${ }^{\dagger}$ Ryota Mukai, ${ }^{\dagger}$ Manabu Ishizaki ${ }^{\ddagger}$ Tomoyuki Toda, ${ }^{\S}$ Masato Kurihara ${ }^{\ddagger}$ and Masaaki Okazaki ${ }^{\dagger}$

\footnotetext{
${ }^{\dagger}$ Department of Frontier Materials Chemistry, Graduate School of Science and Technology, Hirosaki University, 3 Bunkyo-cho, Hirosaki, Aomori 036-8561, Japan

${ }^{\ddagger}$ Faculty of Science, Yamagata University, 1-4-12 Kojirakawa-machi, Yamagata 990-8560, Japan

${ }^{\S}$ Department of Materials Science and Technology, Nagaoka University of Technology, 1603-1

Kamitomioka, Nagaoka, Niigata 940-2188, Japan
}

Contents of Supporting Information

Figure S1. Photograph and schematic of the adsorption experiments

Figures S2-S7. Thermal ellipsoid plots and 3D packings

Figure S8. ${ }^{1}$ H NMR spectrum of crystals of closed form A after exposure to ethanol

Figures S9-S24. ${ }^{1} \mathrm{H}$ NMR spectra of crystals of closed form A after exposure to mixed vapors

Table S1. Crystallographic data 

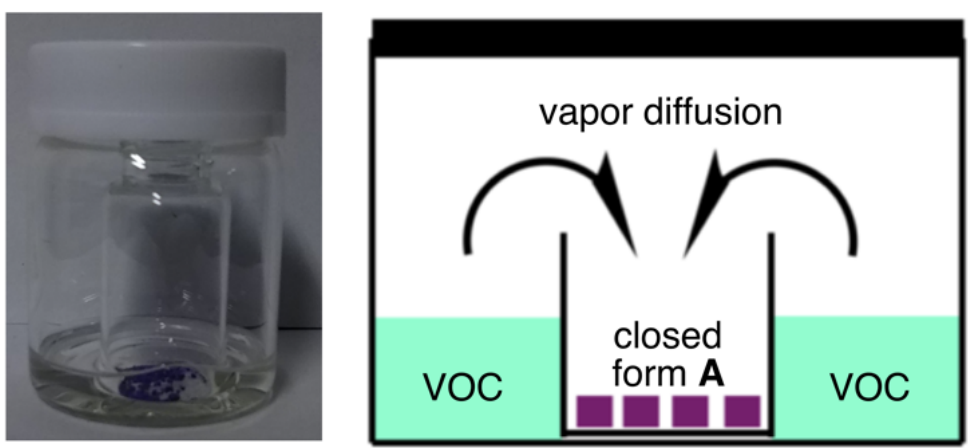

Figure S1. Photograph (left) and schematic illustration (right) of the VOC adsorption experiments with closed form A crystals.

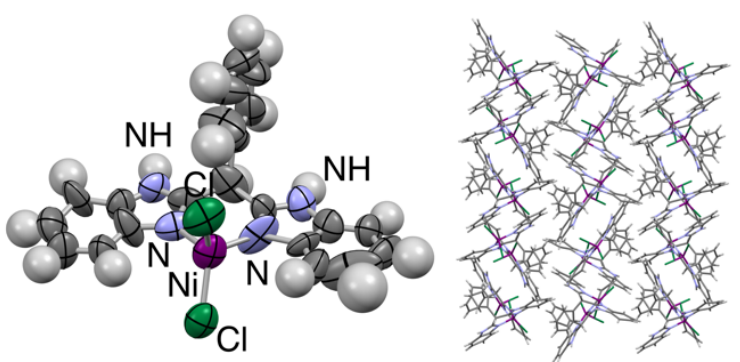

Figure S2. (left) Thermal ellipsoid plot (50\% probability) of $\mathbf{1}$ in crystals of closed form A. (right) 3D packing in crystals of closed form $\mathbf{A}$.


Figure S3. a. (left) Thermal ellipsoid plot (50\% probability) of 1-(THF), which was obtained from exposing crystals of closed form $\mathbf{A}$ to THF vapor. (right) 3D packing of 1.(THF), which was obtained from exposing closed form A crystals to THF vapor. b. (left) Thermal ellipsoid plot (50\% probability) of 1.(THF) obtained from a $\mathrm{CH}_{3} \mathrm{CN} / \mathrm{THF}$ solution of 1. (right) $3 \mathrm{D}$ packing of 1.(THF) obtained from a $\mathrm{CH}_{3} \mathrm{CN} / \mathrm{THF}$ solution of $\mathbf{1}$. 


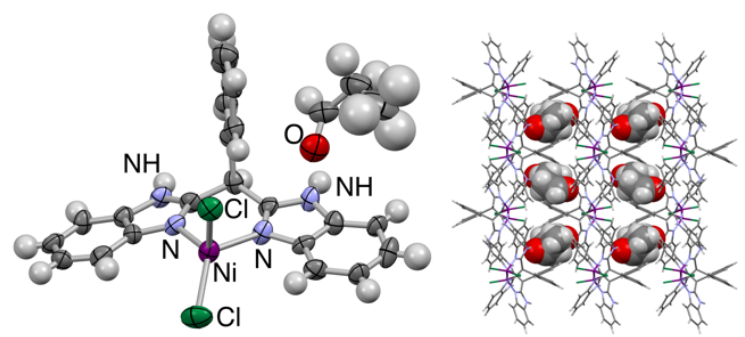

Figure S4. (left) Thermal ellipsoid plot (50\% probability) of $\mathbf{1} \cdot\left(\mathrm{CH}_{3} \mathrm{CH}_{2} \mathrm{CHO}\right)$. (right) 3D packing of $1 \cdot\left(\mathrm{CH}_{3} \mathrm{CH}_{2} \mathrm{CHO}\right)$.

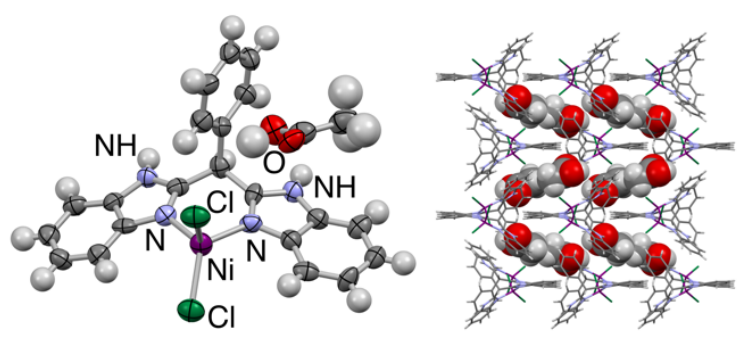

Figure S5. (left) Thermal ellipsoid plot (50\% probability) of $\mathbf{1} \cdot\left(\mathrm{CH}_{3} \mathrm{COOH}\right)$. (right) 3D packing of 1. $\left(\mathrm{CH}_{3} \mathrm{COOH}\right)$.

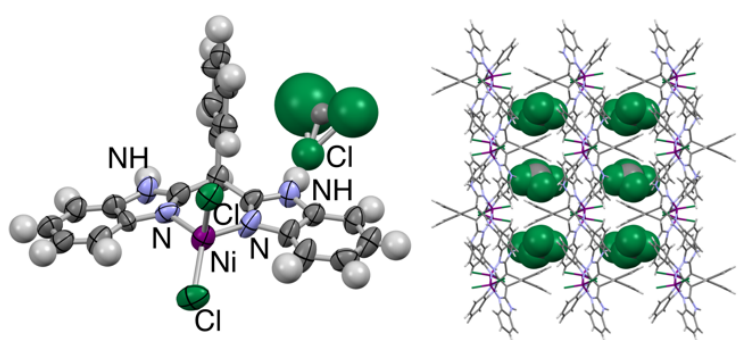

Figure S6. (left) Thermal ellipsoid plot (50\% probability) of $\mathbf{1} \cdot\left(\mathrm{CHCl}_{3}\right)_{0.5} \cdot$ (right) 3D packing of $\mathbf{1} \cdot\left(\mathrm{CHCl}_{3}\right)_{0.5} \cdot$

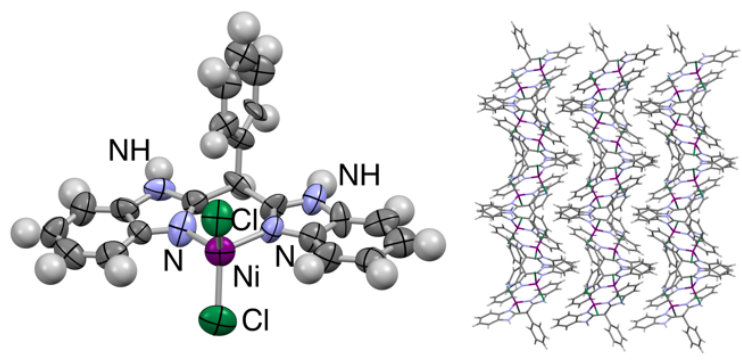

Figure S7. (left) Thermal ellipsoid plot (50\% probability) of $\mathbf{1}$ in the closed form $\mathbf{B}$. (right) 3D packing of the closed form $\mathbf{B}$. 


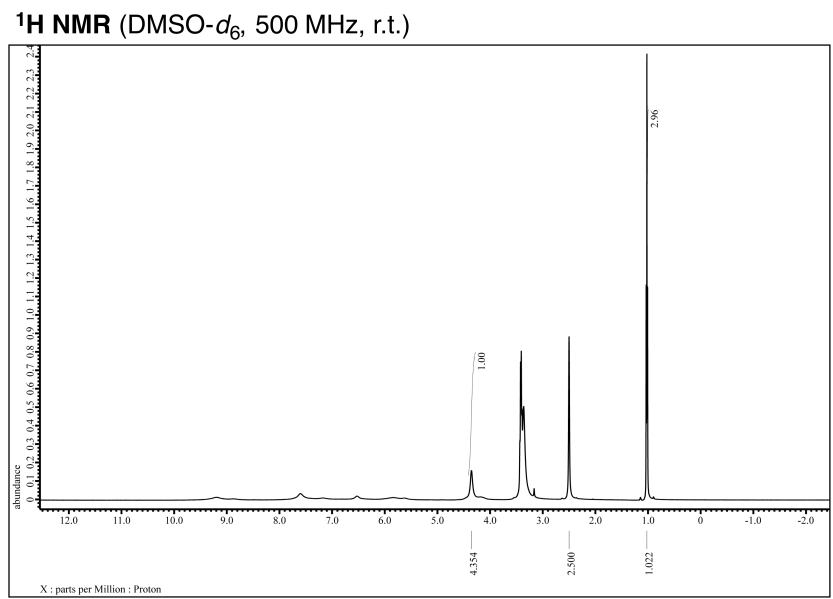

Figure S8. ${ }^{1} \mathrm{H}$ NMR spectrum (DMSO- $d_{6}, 500 \mathrm{MHz}$, r.t.) of crystals of closed form A after exposure to ethanol.

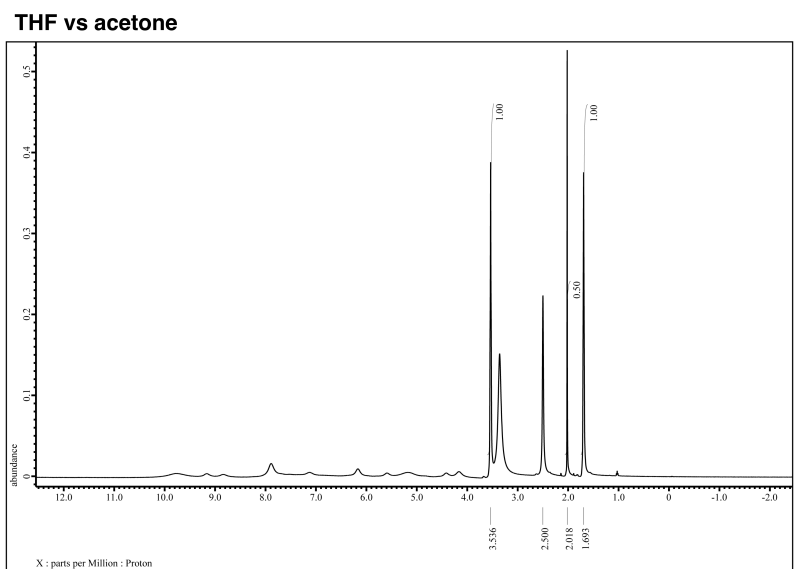

Figure S9. ${ }^{1} \mathrm{H}$ NMR spectrum $\left(\mathrm{DMSO}-d_{6}\right.$, r.t.) of the crystals obtained from exposing closed form A crystals to mixed THF/acetone vapor.

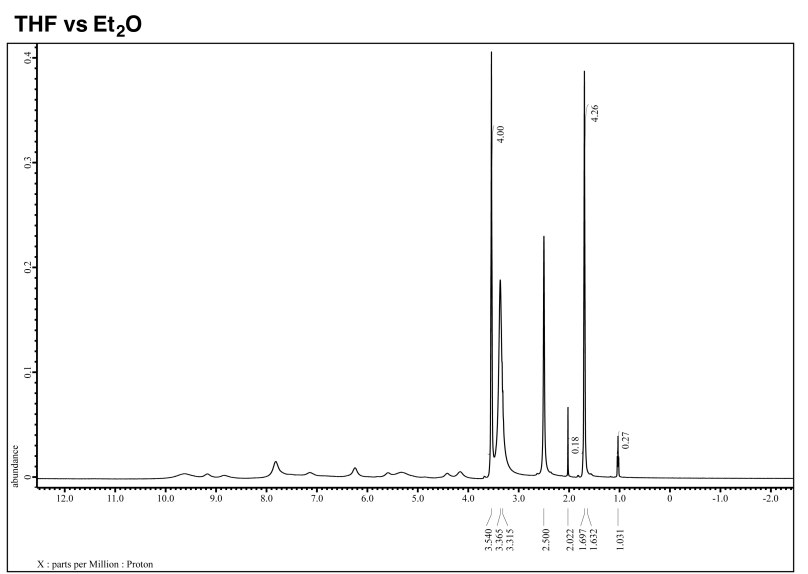

Figure S11. ${ }^{1} \mathrm{H}$ NMR spectrum (DMSO- $d_{6}$, r.t.) of the crystals obtained from exposing closed form $\mathbf{A}$ crystals to mixed $\mathrm{THF} / \mathrm{Et}_{2} \mathrm{O}$ vapor.

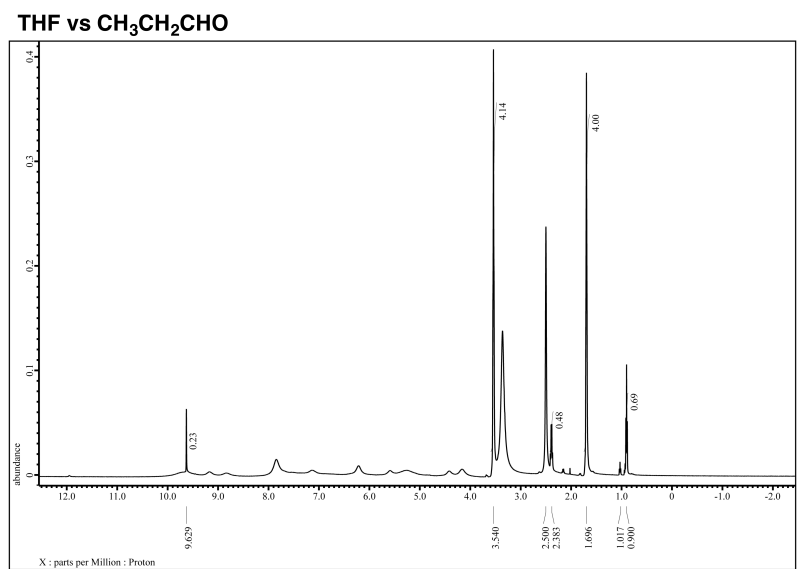

Figure S10. ${ }^{1} \mathrm{H}$ NMR spectrum (DMSO- $d_{6}$, r.t.) of the crystals obtained from exposing closed form A crystals to mixed $\mathrm{THF} / \mathrm{CH}_{3} \mathrm{CH}_{2} \mathrm{CHO}$ vapor.

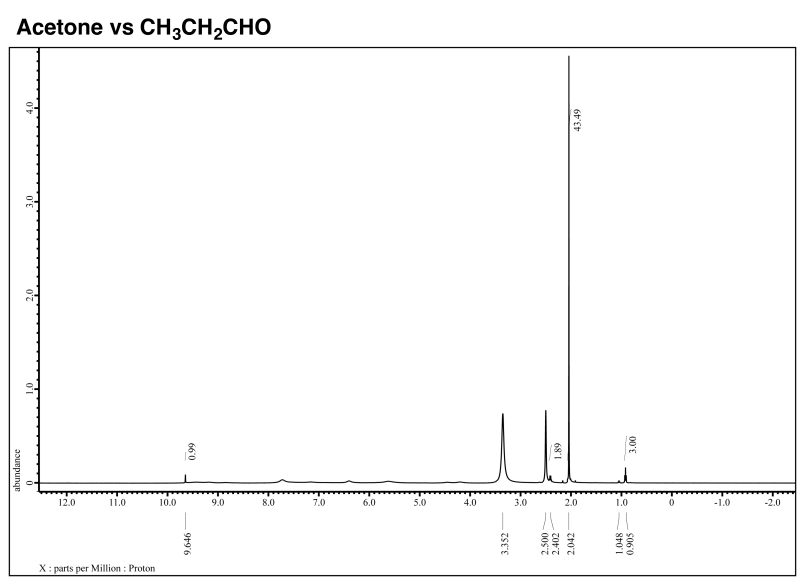

Figure S12. ${ }^{1} \mathrm{H}$ NMR spectrum (DMSO- $d_{6}$, r.t.) of the crystals obtained from exposing closed form A crystals to mixed acetone $/ \mathrm{CH}_{3} \mathrm{CH}_{2} \mathrm{CHO}$ vapor. 


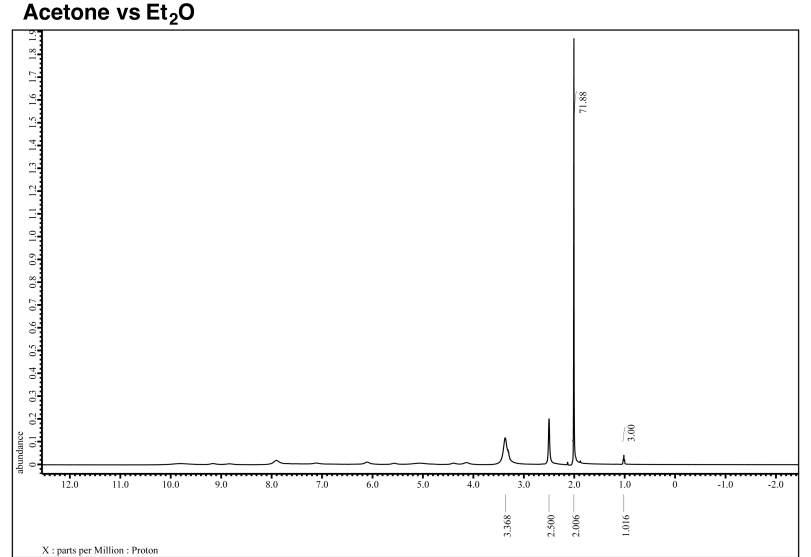

Figure S13. ${ }^{1} \mathrm{H}$ NMR spectrum (DMSO- $d_{6}$, r.t.) of the crystals obtained from exposing closed form $\mathrm{A}$ crystals to mixed acetone $/ \mathrm{Et}_{2} \mathrm{O}$ vapor.

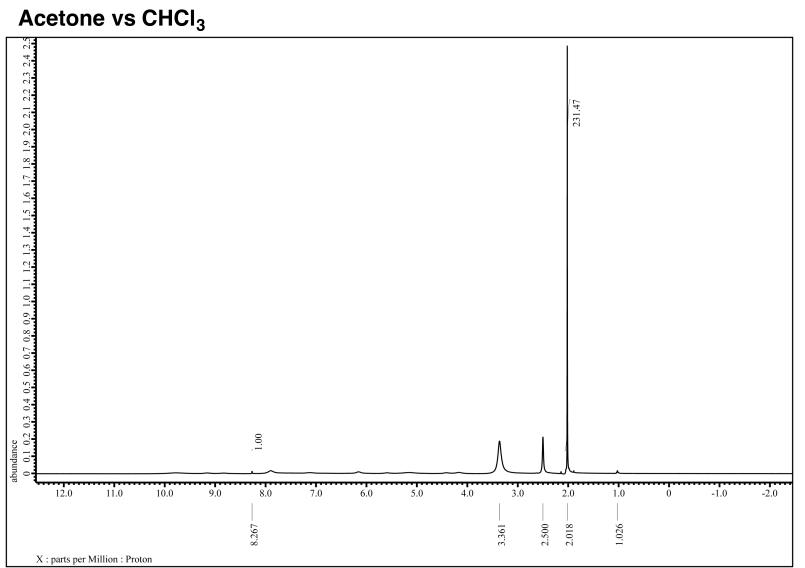

Figure S15. ${ }^{1} \mathrm{H}$ NMR spectrum (DMSO- $d_{6}$, r.t.) of the crystals obtained from exposing closed form $\mathrm{A}$ crystals to mixed acetone $/ \mathrm{CHCl}_{3}$ vapor.

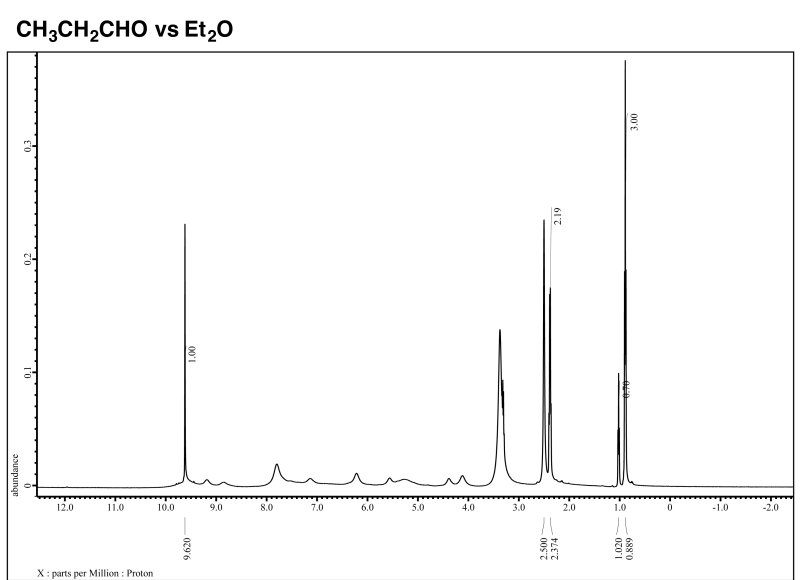

Figure S17. ${ }^{1} \mathrm{H}$ NMR spectrum (DMSO- $d_{6}$, r.t.) of the crystals obtained from exposing closed form A crystals to mixed $\mathrm{CH}_{3} \mathrm{CH}_{2} \mathrm{CHO} / \mathrm{Et}_{2} \mathrm{O}$ vapor.



Figure S14. ${ }^{1} \mathrm{H}$ NMR spectrum (DMSO- $d_{6}$, r.t.) of the crystals obtained from exposing closed form A crystals to mixed acetone/EtOAc vapor.

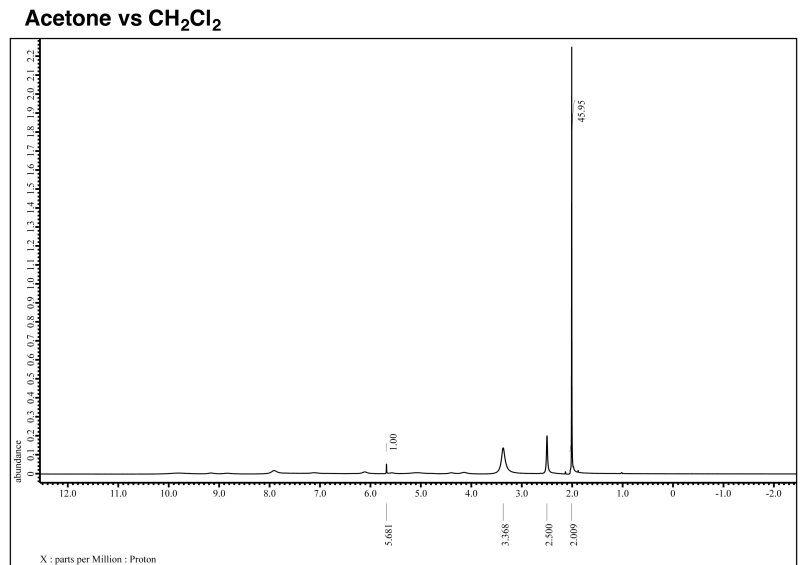

Figure S16. ${ }^{1} \mathrm{H}$ NMR spectrum (DMSO- $d_{6}$, r.t.) of the crystals obtained from exposing closed form $\mathbf{A}$ crystals to mixed acetone $/ \mathrm{CH}_{2} \mathrm{Cl}_{2}$ vapor.

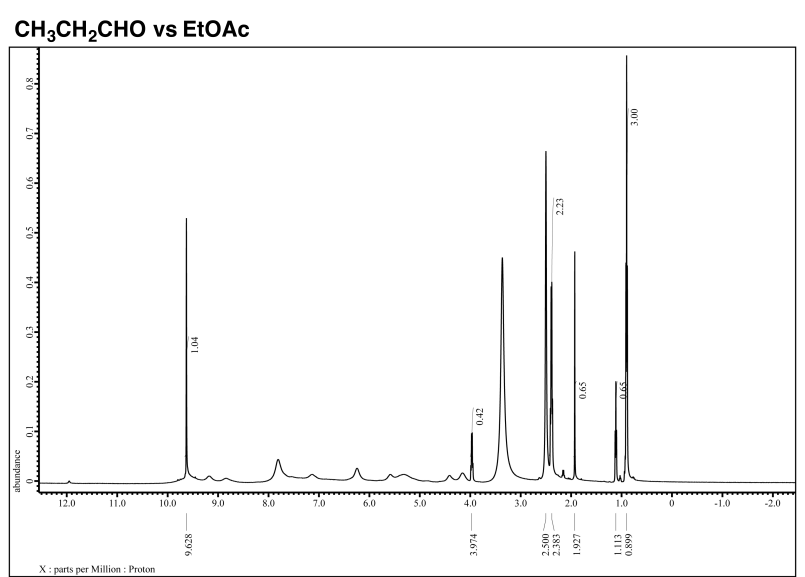

Figure S18. ${ }^{1} \mathrm{H}$ NMR spectrum (DMSO- $d_{6}$, r.t.) of the crystals obtained from exposing closed form A crystals to mixed $\mathrm{CH}_{3} \mathrm{CH}_{2} \mathrm{CHO} / \mathrm{EtOAc}$ vapor. 


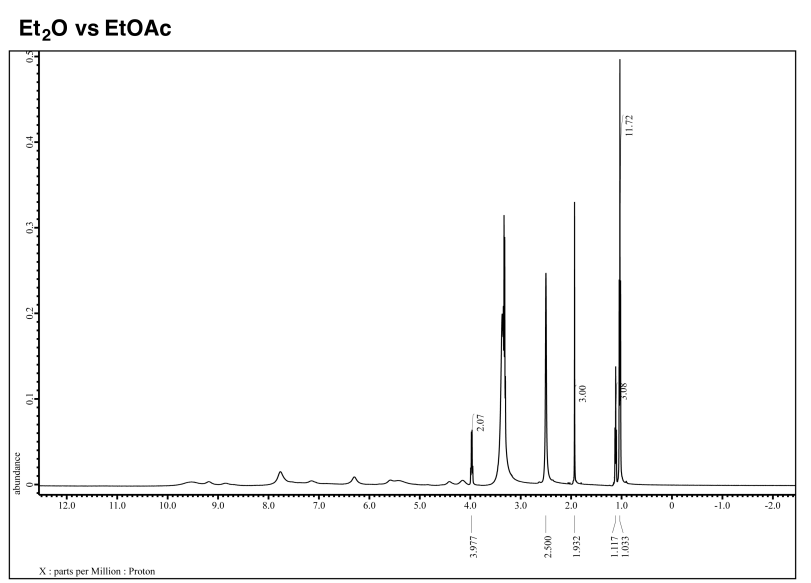

Figure S19. ${ }^{1} \mathrm{H}$ NMR spectrum (DMSO- $d_{6}$, r.t.) of the crystals obtained from exposing closed form $\mathbf{A}$ crystals to mixed $\mathrm{Et}_{2} \mathrm{O} / \mathrm{EtOAc}$ vapor.

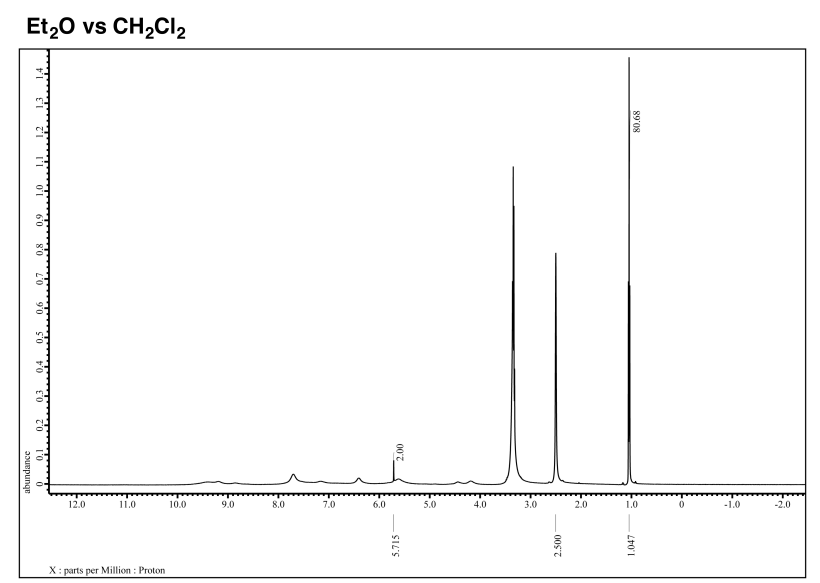

Figure S21. ${ }^{1} \mathrm{H}$ NMR spectrum (DMSO- $d_{6}$, r.t.) of the crystals obtained from exposing closed form $\mathrm{A}$ crystals to mixed $\mathrm{Et}_{2} \mathrm{O} / \mathrm{CH}_{2} \mathrm{Cl}_{2}$ vapor.

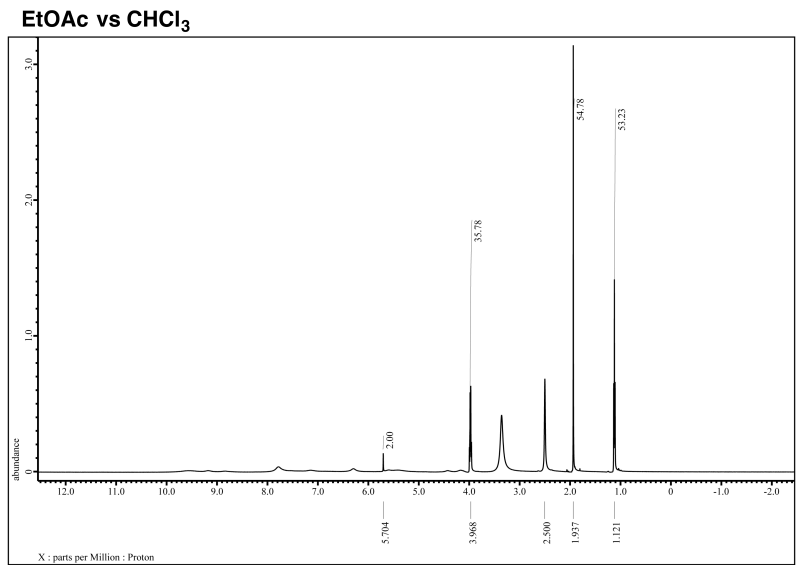

Figure S23. ${ }^{1} \mathrm{H}$ NMR spectrum (DMSO- $d_{6}$, r.t.) of the crystals obtained from exposing closed form A crystals to mixed EtOAc/ $\mathrm{CH}_{2} \mathrm{Cl}_{2}$ vapor.
$\mathrm{Et}_{2} \mathrm{O}$ vs EtOAc

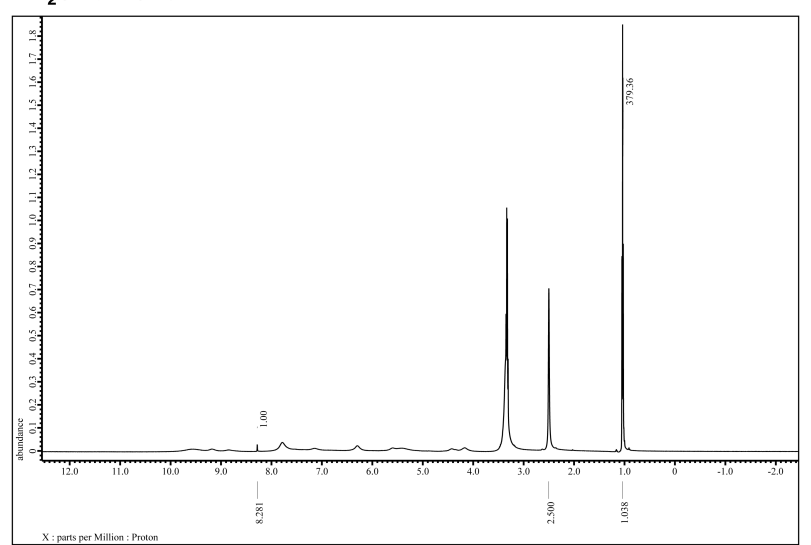

Figure S20. ${ }^{1} \mathrm{H}$ NMR spectrum (DMSO- $d_{6}$, r.t.) of the crystals obtained from exposing closed form $\mathbf{A}$ crystals to mixed $\mathrm{Et}_{2} \mathrm{O} / \mathrm{CHCl}_{3}$ vapor.

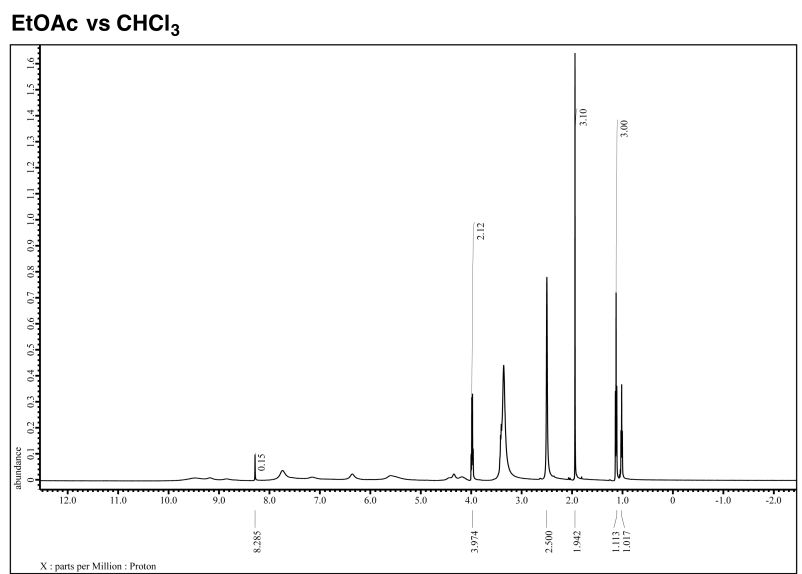

Figure S22. ${ }^{1} \mathrm{H}$ NMR spectrum (DMSO- $d_{6}$, r.t.) of the crystals obtained from exposing closed form $\mathbf{A}$ crystals to mixed EtOAc/ $\mathrm{CHCl}_{3}$ vapor.

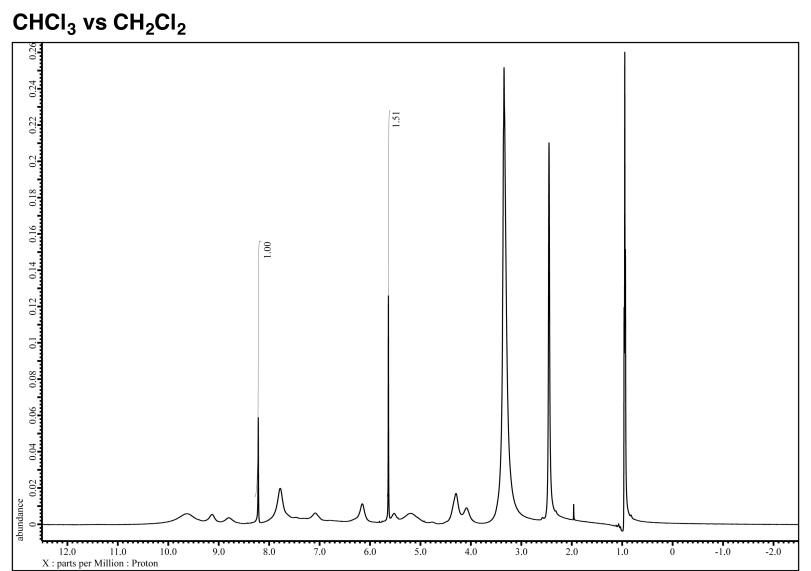

Figure S24. ${ }^{1} \mathrm{H}$ NMR spectrum (DMSO- $d_{6}$, r.t.) of the crystals obtained from exposing closed form $\mathbf{A}$ crystals to mixed $\mathrm{CHCl}_{3} / \mathrm{CH}_{2} \mathrm{Cl}_{2}$ vapor. 
Table S1. Crystallographic data

\begin{tabular}{|c|c|c|c|c|c|c|}
\hline & $\mathbf{1} \cdot\left(\mathrm{Et}_{2} \mathrm{O}\right)$ & Closed form $\mathbf{A}$ & $\mathbf{1} \cdot(\mathrm{THF})^{[\mathrm{a}]}$ & $\mathbf{1} \cdot(\mathrm{THF})^{[\mathrm{b}]}$ & 1. $\left(\mathrm{CH}_{2} \mathrm{Cl}_{2}\right)$ & 1.(acetone) \\
\hline Crystal system & monoclinic & monoclinic & monoclinic & monoclinic & monoclinic & monoclinic \\
\hline Space group & $P 2_{1} / c$ & $P 2_{1} / n$ & $P 2_{1} / c$ & $P 2_{1} / c$ & $P 2_{1} / c$ & $P 2_{1} / c$ \\
\hline$a / \AA$ & $10.7785(7)$ & $16.270(6)$ & $10.176(4)$ & $10.2373(12)$ & $10.2836(19)$ & $9.929(2)$ \\
\hline$b / \AA$ & $14.6846(8)$ & $13.049(5)$ & $14.316(6)$ & $14.3632(14)$ & $13.956(3)$ & $14.250(3)$ \\
\hline$c / \AA$ & $15.8321(8)$ & 19.811(8) & $15.892(6)$ & $16.0660(16)$ & $15.800(3)$ & $16.130(3)$ \\
\hline$\beta / \AA$ & $103.319(7)$ & $107.132(8)$ & $92.844(9)$ & $92.969(6)$ & $95.881(7)$ & $93.218(6)$ \\
\hline$V / \AA$ & $2438.5(3)$ & $4019(3)$ & $2312.2(16)$ & $2359.2(4)$ & $2255.8(7)$ & $2278.6(8)$ \\
\hline$Z$ & 4 & 8 & 4 & 4 & 4 & 4 \\
\hline Goodness of fit & 1.016 & 1.369 & 1.374 & 0.968 & 1.044 & 1.028 \\
\hline$R_{1}(I>2 \sigma(\mathrm{I}))$ & 0.0514 & 0.1966 & 0.2402 & 0.0625 & 0.1022 & 0.1352 \\
\hline $\mathrm{w} R_{2}$ (all data) & 0.0967 & 0.5601 & 0.5933 & 0.1340 & 0.3134 & 0.3622 \\
\hline & $\mathbf{1} \cdot\left(\mathrm{CH}_{3} \mathrm{CH}_{2} \mathrm{CHO}\right)$ & $\mathbf{1} \cdot($ EtOAc $)$ & $\mathbf{1} \cdot\left(\mathrm{CH}_{3} \mathrm{COOH}\right)$ & $\mathbf{1} \cdot\left(\mathrm{CHCl}_{3}\right)_{0.5}$ & \multicolumn{2}{|c|}{ Closed form $\mathbf{B}$} \\
\hline Crystal system & monoclinic & monoclinic & monoclinic & monoclinic & \multicolumn{2}{|c|}{ orthorhombic } \\
\hline Space group & $P 2_{1} / c$ & $P 2_{1} / c$ & $P 2_{1} / c$ & $P 2_{1} / c$ & \multicolumn{2}{|c|}{$P c a 2_{1}$} \\
\hline$a / \AA$ & $10.161(8)$ & $19.314(5)$ & $9.872(2)$ & $10.013(5)$ & \multicolumn{2}{|c|}{$15.814(3)$} \\
\hline$b / \AA$ & $14.346(10)$ & $15.906(4)$ & $15.119(3)$ & $14.441(6)$ & \multicolumn{2}{|c|}{$13.274(3)$} \\
\hline$c / \AA$ & $16.094(11)$ & $16.039(4)$ & $16.205(3)$ & $15.966(6)$ & \multicolumn{2}{|c|}{$19.276(4)$} \\
\hline$\beta / \AA$ & $95.564(16)$ & $90.134(7)$ & $106.477(7)$ & $92.576(12)$ & & \\
\hline$V / \AA$ & $2335(3)$ & $4927(2)$ & 2319.4(8) & $2306.3(17)$ & \multicolumn{2}{|c|}{$4046.3(14)$} \\
\hline$Z$ & 4 & 8 & 4 & 4 & \multicolumn{2}{|c|}{8} \\
\hline Goodness of fit & 1.075 & 1.669 & 1.055 & 1.135 & \multicolumn{2}{|c|}{1.086} \\
\hline$R_{1}(I>2 \sigma(\mathrm{I}))$ & 0.1500 & 0.2166 & 0.1146 & 0.1615 & \multicolumn{2}{|c|}{0.1416} \\
\hline $\mathrm{w} R_{2}$ (all data) & 0.4094 & 0.5919 & 0.3715 & 0.4871 & \multicolumn{2}{|c|}{0.4377} \\
\hline
\end{tabular}

[a] Obtained from exposing closed form A crystals to THF vapor. [b] Obtained from a $\mathrm{CH}_{3} \mathrm{CN} / \mathrm{THF}$ solution of $\mathbf{1}$. 